\title{
Multi-pod structures of lamellae-forming diblock copolymers in three-dimensional confinement spaces: experimental ob- servation and computer simulation
}

Takeshi Higuchi $^{1, *}$, Marco Pinna ${ }^{2, *}$, Andrei V. Zvelindovsky ${ }^{2}$, Hiroshi Jinnai ${ }^{1}$, Hiroshi Yabu ${ }^{1,3, *}$

${ }^{1}$ Institute of Multidisciplinary Research for Advanced Materials (IMRAM), Tohoku University, 21-1, Katahira, Aoba-ku, Sendai 980-8577, Japan

${ }^{2}$ School of Mathematics and Physics, University of Lincoln, Brayford Pool, Lincoln, LN6 7TS, United Kingdom

${ }^{3}$ Precursory Research for Embryonic Science and Technology, Japan Science and Technology Agency, Research Division Gobancho Building 5F, 7 Gobancho, Chiyoda-ku, Tokyo 102-0076, Japan

Correspondence to: Takeshi Higuchi (E-mail: higuchi@tagen.tohoku.ac.jp), Marco Pinna (E-mail: mpinna@lincoln.ac.uk), Hiroshi Yabu (E-mail: yabu@tagen.tohoku.ac.jp)

Dated: March 29, 2016

\begin{abstract}
The three-dimensional (3D) confinement effect on the microphase-separated structure of a diblock copolymer was investigated both experimentally and computationally. Block copolymer nanoparticles were prepared by adding a poor solvent into a block copolymer solution and subsequently evaporating the good solvent. The 3D structures of the nanoparticles were quantitatively determined with transmission electron microtomography (TEMT). TEMT observations revealed that various complex structures, including tennis-ball, mushroom-like, and multi-pod structures, were formed in the $3 \mathrm{D}$ confinement. Detailed structural analysis, showed that one block of the diblock copolymer slightly prefers to segregate into the particle surface compared with the other block. The observed structures were further elaborated using cell dynamics computer simulation.
\end{abstract}

Keywords: Block copolymer, Microphase-separated strucuture, 3D confinement, Electron tomography, Cell Dynamic Simulation 


\section{INTRODUCTION}

Block copolymers consist of covalently bonded dissimilar polymer segments and can spontaneously form periodic microphase-separated structures. The characteristic lengths of the structures depend on their molecular weights and are generally in the range from 10 to 100 $\mathrm{nm}$. The typical morphologies of diblock copolymers include spheres, cylinders, gyroids, and lamellae, depending on their volume fraction. Highly ordered nanostructures can be prepared by simple coating techniques, thus microphase-separated structures have been developed for diverse applications, for example, etching masks in lithography, ${ }^{1}$ photonic crystals ${ }^{2}$ filtration, ${ }^{3}$ and photovoltaic devices. ${ }^{4}$ The performance of these applications is governed by the morphologies of the microphase-separated structures ${ }^{5}$ so control of morphologies and exploration of novel structures are challenging.

Recent developments in polymerization techniques have revealed the diversity of the molecular structures of block copolymers, including grafts, stars, multiblocks, gradients, and tapered block sequences. ${ }^{6-10}$ Consequently, various novel morphologies have been reported. ${ }^{11-15}$ The nonsynthetic way to create novel morphologies is to confine block copolymers into nanospaces. Block copolymers in confinements form unique morphologies known as "frustrated phases", which are different from the morphologies in the bulk. The frustrated phases receive much attention in both theoretical ${ }^{16}$ and experimental ${ }^{17}$ science communities. Various types of confinement spaces have been used, including one-dimensional confinement, 1D (thin films), ${ }^{18-21}$ two-dimensional, 2D (e.g., cylindrical pores of anodized aluminum oxide membranes), ${ }^{22-24}$ and three-dimensional, 3D (e.g., spherical pores of inverse colloid crystals) ${ }^{25-27}$ confinements. Morphologies in a confinement are mainly governed by three parameters: i) the size of the confinement, ii) its shape, and iii) the interfacial energy between the polymer and outer matrix. When the size of the confinement space is not a multiple integer of the natural period of the microphase-separated structures in bulk, unique morphologies are formed due to frustration in order to accommodate periodic domains in confinement spaces. Therefore, the confined structures will depend on $D / L_{0}$, where $D$ is the size of the confinement space and $L_{0}$ is the period of the microphase-separated structure in the bulk sample. The surface property of a confinement space is another important factor 
that determines frustration. Chemical modification and topological patterning of the surfaces are often used to control the affinity of the polymer blocks towards the surface. ${ }^{28-30}$ A fascinating example is the formation of helical domains from diblock copolymers in cylindrical pores with a non-integer period size, even though the block copolymer has no chiral center in the molecular structure. ${ }^{22}$

Previously, we reported that diblock copolymers consisting of polystyrene and polyisoprene blocks form various nanoparticle morphologies in 3D confinement. ${ }^{31-33}$ Although the morphologies of the nanoparticles were too complex to characterize them by conventional transmission electron microscopy (TEM), the 3D structures were quantitatively analyzed with transmission electron microtomography (TEMT). ${ }^{34}$ TEMT revealed that helical domains were formed on the surfaces of the nanoparticles, which are frustrated phases induced by the $3 \mathrm{D}$ confinement effect. Several theoretical groups have simulated the morphologies of diblock copolymers in a 3D confinement. ${ }^{35-39}$. In Ref. 36, one of the coauthors of the present paper has investigated diblock copolymer in a solvent by means of dynamic self-consistent field theory (SCFT). The effect of diblock copolymer composition as well as interaction strength between the polymer segments and the solvent on the observed morphologies has been studied. A rich "zoo" of structures has been found, such as onions, sandwich lamellae particles, particles with tori inside, as well as more exotic internal structures such as "red blood cells" plateletes and coffee beans. The work also predicted formation of patched nanoparticles (see Fig. 3 right in Ref. 36). Later Shi and collaborators group has studied symmetric and asymmetric diblock copolymers as well as binary blends of diblock copolymer and homopolymer in a 3D confinement using simulated annealing methods and SCFT. ${ }^{37,38,40,41}$ More recently the study was extended using simulated annealing method to patchy spherical nanoparticles self-assembled from linear ABC triblock copolymers. ${ }^{42}$ To date a direct comparison between experiment and simulation is very limited. ${ }^{39}$ A detailed comparison between experimental and theoretical results can lead to further understanding of the parameters governing formation of frustrated morphologies and eventually to a computer-aided design of specific tailored structures.

In the present work, we report on a joint experimental and computer simulation study of the frustrated phases of ultra high molecular weight block copolymers in 3D spherical 
confinement. Block copolymer nanoparticles were prepared by adding a poor solvent into a block copolymer solution and subsequently evaporating the good solvent. The microphaseseparated structures of nanoparticles were determined with TEMT. The same structures were obtained using cell dynamics computer simulation.

\section{EXPERIMENTS}

\section{Preparation of block copolymer nanoparticles}

A block copolymer with polystyrene and polyisoprene segments (PSt- $b$-PI: $M_{n(P S t)}=700,000$, $\left.M_{n(P I)}=850,000, f_{P I}=0.58, M_{w} / M_{n}=1.15\right)$, purchased from Polymer Source Inc. Ltd., Canada, was used in this work. The polymer was dissolved in tetrahydrofuran (THF with a stabilizer, Wako Pure Chemical Industries, Ltd., Japan) at a concentration of $0.1 \mathrm{~g} / \mathrm{L}$. Deionized water $(2 \mathrm{~mL})$ was added to $1 \mathrm{~mL}$ of the block copolymer solution with stirring. The block copolymer was then precipitated as nanoparticles with the gradual evaporation of $\mathrm{THF}$ at $25^{\circ} \mathrm{C}$ under atmospheric pressure for more than 2 days.

\section{Observation of phase-separated structures in block copolymer nanoparticles with TEM and TEMT}

To observe phase-separated structures in block copolymer nanoparticles with TEM, $0.2 \mathrm{~mL}$ of nanoparticle dispersions were stained with $0.2 \mathrm{~mL}$ of $0.2 \mathrm{wt} \% \mathrm{OsO}_{4}$, which selectively reacts with double bonds in polyisoprene segments, for $2 \mathrm{~h}$ at room temperature. After staining, particles were collected by centrifugation $\left(12,000 \mathrm{rpm}, 5^{\circ} \mathrm{C}, 15 \mathrm{~min}\right)$ and washed twice with deionized water to remove excess $\mathrm{OsO}_{4}$. After washing, the stained particles were re-dispersed in deionized water with ultrasonication. The dispersion of the stained particles $(2 \mu \mathrm{L})$ was placed onto the surface of a polyvinyl formal membrane placed on a $\mathrm{Cu}$ mesh and dried at room temperature. The phase-separated structures in the particles were observed using a scanning transmission electron microscopy (STEM, S-5200 equipped with a TEM detector, Hitachi Ltd., Japan) operated at 40 kV. For TEMT observations, gold nanoparticles ( $5 \mathrm{~nm}$ diameter) were deposited on the bottom of the supporting membrane. A series of TEM images were acquired at tilt angles of around $\pm 70^{\circ}$ at an angular interval of $1^{\circ}$ 
with a JEM-2200FS microscope (JEOL, Ltd., Japan) operated at $200 \mathrm{kV}$ and equipped with a slow-scan USC 4000 CCD camera (Gatan, Inc., USA). The angular range is sufficient for quantitative analysis. ${ }^{43}$ The tilt series of the TEM images were then aligned by the fiducial marker method, using gold nanoparticles as the markers. ${ }^{44}$ The tilt series of TEM images after alignment were reconstructed by filtered back projection. ${ }^{45,46}$

\section{Area ratios occupied by $\mathrm{PI}$ phases at the particle surfaces measured from 3D structures}

To evaluate interfacial energy between polymer segments of the block copolymer and an external matrix (i.e., water), the area ratios occupied with PI phases at the particle surfaces were experimentally measured from 3D data obtained with TEMT. The surface areas of PSt and PI phases were individually measured from their 3D data, and were defined as $A_{P S t}$ and $A_{P I}$. Each surface area was composed of two interfaces: polymer/water $\left(A_{P S t / w a t e r}\right.$ and $\left.A_{P I / \text { water }}\right)$ and PSt/PI interfaces $\left(A_{P S t / P I}\right)$. The relationship between the surface areas of particles $\left(S_{P}\right)$ and these interfacial areas can be described as follows:

$$
\begin{aligned}
A_{P S t}=A_{P S t / w a t e r}+A_{P S t / P I} & , A_{P I}=A_{P I / \text { water }}+A_{P S t / P I} \\
S_{P} & =A_{P S t / \text { water }}+A_{P I / \text { water }} \\
& =A_{P S t}+A_{P I}-2 A_{P S t / P I}
\end{aligned}
$$

Because it is difficult to directly measure $A_{P S t / P I}$ from $3 \mathrm{D}$ data, the area ratios occupied by PI phases at the particle surfaces $\left(S_{P I}\right)$ can be calculated as follows:

$$
\begin{aligned}
S_{P I} & =\frac{A_{P I / \text { water }}}{S_{P}} \\
& =\frac{A_{P I}-A_{P S t / P I}}{S_{P}} \\
& =\frac{A_{P I}-A_{P S t}-S_{P}}{2 S_{P}}
\end{aligned}
$$

\section{Simulation of phase-separated structures in block copolymer nanoparticles}

In the present work, we used cell dynamics simulation (CDS) to study the block copolymer morphologies in a spherical confinement. CDS is a very fast computational technique ${ }^{47-50}$ and can serve as a precursor for the computer-aided design of block copolymer systems. For $A B$ diblock copolymers, the structure can be described by an order parameter $\psi(\mathbf{r}, t)$ : 


$$
\psi=\phi_{A}-\phi_{B}+(1-2 f)
$$

where $\phi_{A}$ and $\phi_{B}$ are the local volume fractions of $A$ and $B$ monomers respectively, and $f$ is the volume fraction of $A$ monomers in the diblock, $f=N_{A} /\left(N_{A}+N_{B}\right)$ with $N_{i}$ signifying the number of $i$-monomers. The time evolution of the order parameter is given by a CahnHilliard-Cook equation:

$$
\frac{\partial \psi}{\partial t}=M \nabla^{2}\left(\frac{\delta F[\psi]}{\delta \psi}\right)
$$

where $M$ is a phenomenological mobility constant. The dimensionless time is $t M / a_{0}^{2}$, where the lattice cell size $a_{0}$, and $M$ are set to 1 . The free energy functional is ${ }^{51}$

$$
\begin{aligned}
F[\psi(\mathbf{r})] & =\int d \mathbf{r}\left[H(\psi)+\frac{D}{2}|\nabla \psi|^{2}\right] \\
& +\frac{B}{2} \int d \mathbf{r} \int d \mathbf{r}^{\prime} G\left(\mathbf{r}-\mathbf{r}^{\prime}\right) \psi(\mathbf{r}) \psi\left(\mathbf{r}^{\prime}\right)
\end{aligned}
$$

where

$$
H(\psi)=\left[-\frac{\tau}{2}+\frac{A}{2}(1-2 f)^{2}\right] \psi^{2}+\frac{v}{3}(1-2 f) \psi^{3}+\frac{u}{4} \psi^{4}
$$

with $\tau A, v, u, D, B$ being phenomenological parameters, and $G\left(\mathbf{r}-\mathbf{r}^{\prime}\right)$ is the Green ' $\mathrm{s}$ function for the Laplace equation. All these parameters can be related to molecular characteristics. According to Ohta and Kawasaki ${ }^{51} \tau^{\prime}=-\tau+A(1-2 f), D$, and $B$ can be expressed in terms of degree of polymerization $N$, the segment length $b$ and the Flory-Huggins parameter $\chi_{\complement}$-In simulation we use dimensionless parameters $\tilde{D}=D / a_{0}^{2}$ and $\tilde{B}=B / a_{0}^{2}$ (for simplicity we use notations $D$ and $B$ instead of $\tilde{D}$ and $\tilde{B}$ ). Different morphologies (lamellae, spherical, cylindrical) can be obtained by varying two main controlling parameters, that is, the molecular compositions $f$ and the temperature like-parameter $\tau^{\prime}$. The parameters $u$ and $v$ do not allow for a compact representation; they can be computed by evaluating the appropriate vertex functions given by Leibler. ${ }^{52}$ These are very complex functions that can be replaced only approximately by constants. 
The numerical evolution of Eq. 5 by means of cell dynamics simulation is given by: ${ }^{53,54}$

$$
\begin{aligned}
\psi(\mathbf{n}, t+1) & =\psi(\mathbf{n}, t)-\{\langle\langle\Gamma(\mathbf{n}, t)\rangle\rangle \\
& -\Gamma(\mathbf{n}, t)+B \psi(\mathbf{n}, t)\}
\end{aligned}
$$

where $\langle\langle X\rangle\rangle-X$ is the isotropized discrete Laplacian for quantity $\mathrm{X},{ }^{47} \mathbf{n}=\left(\mathrm{n}_{x}, \mathrm{n}_{y}, \mathrm{n}_{z}\right)$ and

$$
\begin{aligned}
\Gamma(\mathbf{n}, t) & =g(\psi(\mathbf{n}, t))-\psi(\mathbf{n}, t) \\
& +D[\langle\langle\psi(\mathbf{n}, t)\rangle\rangle-\psi(\mathbf{n}, t)]
\end{aligned}
$$

where the so-called map function is: ${ }^{48,55}$

$$
g(\psi)=\left[1+\tau-A(1-2 f)^{2}\right] \psi-v(1-2 f) \psi^{2}-u \psi^{3}
$$

The formula

$$
\langle\langle\psi\rangle\rangle=\frac{6}{80} \sum_{\mathrm{NN}} \psi+\frac{3}{80} \sum_{\mathrm{NNN}} \psi+\frac{1}{80} \sum_{\mathrm{NNNN}} \psi
$$

is used to calculate the isotropized Laplacian $\langle\langle X\rangle\rangle-X$; the discrete lattice is shown in Fig. 1.

We chose the simplest model, in which the preference of copolymer blocks to the surface is described by the Dirichlet boundary condition $\psi(r=R)=\psi_{0} \cdot{ }^{56}$ The spherical surface is approximated on the discrete Cartesian lattice with values $\psi_{0}$ assigned to the order parameter on the lattice point outside of a chosen radius $R$. A typical range for $\psi_{0}$ is reported in our earlier works ${ }^{24,39}$ on diblock copolymers confined in a cylindrical and spherical confinements. In the present work we chose $\psi_{0}$ value to match a specific experimental structure. All simulations were carried out for 200,000 time steps with $\tau=0.40, B=0.02, A=1.6$, $D=0.7, u=0.38, v=0.46$ (see the references ${ }^{57,58}$ for CDS phase diagram). The bulk domain spacings between lamellae were found to be $L_{0} \cong 7.1$ grid points.

\section{RESULTS and DISCUSSION}

Block copolymer nanoparticles were prepared via self-organization processes (Fig. 2). ${ }^{59}$ To observe the microphase-separated structures with TEM, the block copolymer nanoparticles were stained with $\mathrm{OsO}_{4}$, which selectively reacts with double bonds in the polyisoprene 
segments. As shown in the scanning transmission electron microscopy (STEM), Fig. 2, non-lamellar structures are formed in the nanoparticles, even though this block copolymer forms lamellae in bulk samples.

TEM images of PSt- $b$-PI nanoparticles are shown in Fig. 3(a1)-(a6) for different ratios of particle diameter $D$ to the natural period of lamellae in the bulk, $L_{0}$. The bright and dark regions of the nanoparticles depict the unstained PSt moieties and the PI moieties stained with $\mathrm{OsO}_{4}$, respectively. To determine the 3D structures of the microphase-separated structures in PSt- $b$-PI nanoparticles, 3D structural analysis was carried out with TEMT. The 3D structures of the PSt and PI phases are shown separately in Fig. 3(b1)-(b6) and (c1)(c6). The blue and green regions correspond to the PSt and PI phases, respectively. When the ratio $D / L_{0}$ was lower than 0.62 , a spherical particle with a biphasic structure (i.e., a Janus structure) was formed. A Janus structure is the simplest lamellar structure, it forms in a nanoparticle because the particle size is close to the ideal thickness of a single layer of block copolymers $\left(\approx 0.5 L_{0}\right)$. 3D structural analysis with TEMT revealed that the block copolymer formed curved PSt and PI phase interfaces for larger $D / L_{0}$ values, even though block copolymer would naturally prefer to form flat interfaces for this molecular composition. When the $D / L_{0}$ is 0.82 , a mushroom-like PI phase is observed (Fig. 3(b3)), which can be considered as a Janus-derived structure, because a mushroom incorporates a hemisphere and a pod. As $D / L_{0}$ increases to 0.85 , a tennis-ball like structure is formed, which is an another Janus-like structure with a curved interface between the PSt and PI phases (Fig. 3(c3)). When the $D / L_{0}$ becomes 1.02 , a single pod is added to the tennis ball-like structure in the PI phase (Fig. 3(d3)). The number of pods (indicated by numbers in square brackets in Fig. 3) increases with $D / L_{0}$ : PI phase exhibits a tennis-ball like structure with mono- (Fig. 3(d3)), di- (Fig. 3(e3)), tri- (Fig. 3(f3)), tetra- (Fig. 3(g3)), and penta- (Fig. 3(h3)) pods. This morphological series indicates that the area of the curved interfaces between PSt and PI increases with $D / L_{0}$. On the other hand, the volume fraction of the PI phase $\left(f_{\mathrm{PI}}\right)$ as measured from the $3 \mathrm{D}$ data is $0.60 \pm 0.01$, which is similar to that in the bulk state (i.e., 0.58). Thus, the density of polymers in the nanoparticles is similar to that in the bulk state. These results suggest that the block copolymer might respond to the $3 \mathrm{D}$ confinement not by changing the molecular conformation but by the formation of curved interfaces inside the 
nanoparticles.

In the case of the largest particles, the spherical PSt domains were formed as indicated by the white arrow in Fig. 3(h2). The distance from the surface of the central PSt domain to the surfaces of the particles corresponds to $0.52 L_{0}$, which is close to a single layer of lamellar structure $\left(\approx 0.5 L_{0}\right)$. We have previously reported similar structures in PSt- $b$ PI nanoparticles with different volume fractions and molecular weights compared to the block copolymer used in the present experiments. ${ }^{34}$ In our previous work complex structures were formed in the region of a single layer of lamellar structure of the block copolymer $(\approx$ $\left.0.5 L_{0}\right)$, whereas spherical domains were formed for the single-molecule length region. Similar structures were observed for different block copolymers, suggesting a generic behaviour in the $3 \mathrm{D}$ confinement.

The interfacial energy between the polymer blocks and the surrounding dispersion medium is another important factor to influence microphase-separated structures inside the nanoparticles. We can analyze this effect by considering the block copolymer structures at the surface of nanoparticles. The surface of all nanoparticles were composed of both polymer phases (Fig. 3). To evaluate the effect of interfacial energy, the area fraction occupied by PI phase at the particle surface $\left(S_{\mathrm{PI}}\right)$ were measured from the 3D data (Fig. 4). In the case of Janus structures (Fig. 3 (a2), (a3)), $S_{\mathrm{PI}}$ is found to be 0.59 , which is close to the segment ratio of a block copolymer molecule $\left(f_{\mathrm{PI}}=0.58\right)$. This proximity of the two values is easy to understand as all junction points of block copolymer molecules in a Janus particle are located on a single plane interface, and both PSt and PI segments are exposed to the particle outer surface. The area occupied by PI blocks $S_{\mathrm{PI}}$ slightly decreases with increasing $D / L_{0}$ value, which suggests that PSt segments have a slight preference to the water/polymer interface compared to PI segments. For small particles, their internal structure is predominantly influenced by the confinement effect. With the particle size increasing the influence of confinement on the morphology decreases, whereas the effect of the interfacial energy becomes more important. As a result the surface area occupied by PSt phase grows due to a small difference in surface segregation preference between PSt and PI segments.

To compare the experimental and theoretical results, the structures in the $3 \mathrm{D}$ confinement spaces were determined using CDS. The $f_{\mathrm{PI}}$ is chosen to match the experimental data. 
From the experiment is know that PSt segments prefer the outer surface of the particle. Therefore we chose the boundary condition $\psi_{0}=0.2$ in order to mimic that experimental behaviour and more specifically to match the experimental structures in Fig. 3(h). The simulation results are shown in Fig. 5 and 6 . When the volume fraction $f_{\mathrm{PI}}=0.60$ and $D / L_{0}=1.7$, Fig. 5(a)), we found a mushroom-like structures, which is very similar to the experimental structure in Fig. 3(b). For $D / L_{0}=1.8$ we found that the structure of the minor component is similar to a toroid, while the major component forms a cylinder in its middle (see Fig. 5(b)). By increasing $D / L_{0}$ we found tennins-ball morphologies with di-pod (Fig. 5(c)), tri-pod (Fig. 5(d)), tetra-pod (Fig. 5(e)) and penta-pod structures (Fig. 5(f)) corresponding to the experimental results shown in Fig. 3(e3),(f3),(g3), and (h3), respectively. The structure in Fig. 5(f) is very similar to the experimental one in Fig. 3(h) which contains a small sphere in the middle of the minor component (PSt). As we see from the experimental results in Fig. 3 the effective content of PI phase varies between 0.58 till 0.61 . That will influence the affinity of PSt component to the outer surface of the particle. From our simulations we find that by decreasing this affinity we can achieve an even better agreement with the experiments for smaller particle sizes. For $D / L_{0}=1.5,1.7$ and 2.1 we found Janus particle, tennis ball, and a single pod within the tennis ball respectively (Fig. 6) which are very similar to the experimental structures in $3(\mathrm{a}),(\mathrm{c}),(\mathrm{d})$. The results demonstrate a remarkable agreement between the experiments and simulations for various confinement sizes and block copolymer compositions. The results show the identical sequence of structures in both experiments and simulations, which demonstrates a potential to predict the morphologies. The results imply that the assumption of a PSt segment being slightly attracted to the polymer/water interfaces is correct, and the simulation conditions accurately reflect the experimental system. The exact values of $D / L_{0}$ differ in the simulation and experiment, which is likely due to the fact that the model boundary condition on the hard sphere oversimplifies the experimental situation where a polymer particle is immersed in a solvent. However, our results quantitatively agree with the earlier simulated annealing results, which used less simple boundary condition at the particle surface. ${ }^{40}$ For instance, our structure in Fig. $5(\mathrm{~b})$ at $D / L_{0}=1.8$ can be also found in simulated annealing results in Ref. 40 (see their Fig. $1\left(\right.$ a) at $D / L_{0}=1.6$ ). We note that a complete comparison is 
not possible as the simulated annealing paper ${ }^{40}$ uses symmetric diblock copolymer while our study uses asymmetric diblock copolymer. The ideally symmetrical diblock copolymers dislike to form curved interfaces such as sphere as they intrinsically form planer interfaces. In fact, it is pretty difficult (or near impossible) to synthesize the ideal symmetrical molecular structure because an actual block copolymers have always slight deviations in volume fraction of polymer segments as well as molecular weight distribution for each polymer segment. The diblock copolymer used in this study has a slightly larger PI volume $\left(f_{\mathrm{PI}}=0.58\right)$. Such a small asymmetry has little effect on the morphology in the bulk samples whereas it can be critical to break the structural symmetry in 3D confinement. Therefore, we performed simulations reflecting the experimental condition. Our results confirm similar tendencies to form multipod structures in other systems. Yu et al. have reported the multi-pods structures analogous to our experimental results for liner ABC triblock copolymers in spherical confinement using simulated annealing. ${ }^{42}$ It should also be mentioned however, that the structure of the largest particle in the present study, which is shown in Fig. 3(h2, h3) and 5(f1, f2), has not been found in other simulations.

\section{CONCLUSIONS}

We have investigated the frustrated phases of a lamellar-forming diblock copolymer inside spherical confinements using complementary experimental and computer simulation approaches. The block copolymer nanoparticles were prepared by a self-organization process, their 3D structures were quantitatively resolved with TEMT. In small confinement spaces the block copolymer formed Janus-type nanoparticle structures. For larger confinement diameters various complex structures such as tennis-ball, mushroom and multi-pod structures were found. TEMT observations revealed that PSt polymer segments have a slight preference to segregate at the particle surfaces. All experimentally observed morphologies were reproduced by cell dynamics computer simulation. This paper suggests a tandem approach in designing new structures in confinement spaces: computer simulations can be used in order to predicted block copolymer structures in confinements prior to real experiments. 


\section{ACKNOWLEDGMENTS}

This work has been partially supported by JSPS KAKENHI Grants no. 25706006, 26708025 and 24310092, Japan. 


\section{References}

1. M. Park, C. Harrison, P. M. Chaikin, R. A. Register, D. H. Adamson, Science 1997, 276, 1401-1404.

2. C. Kang, E. Kim, H. Baek, K. Hwang, D. Kwak, J. Am. Chem. Soc. 2009, 131, 7538 7539 .

3. S. Yang, I. Ryu, H. Kim, J. Kim, S. Jang, T. P. Russell, Adv. Mater. 2006, 18, 709-712.

4. K. Johnson, Y.-S. Huang, S. Huettner, M. Sommer, M. Brinkmann, R. Mulherin, D. Niedzialek, D. Beljonne, J. Clark, W. T. S. Huck, R. H. Friend, J. Am. Chem. Soc. 2013, 135, 5074-5083.

5. C. Park, J. Yoon, E. L. Thomas, Polymer 2003, 44, 6725-6760.

6. N. Hadjichristidis, H. Iatrou, M. Pitsikalis, S. Pispas, A. Avgeropoulos, Prog. Polym. Sci. 2005, 30, 725-782.

7. S. Jouenne, J. A. Gonzalez-Leon, A.-V. Ruzette, P. Lodefier, S. Tence-Girault, L. Leibler, Macromolecules 2007, 40, 2432-2442.

8. S. Qin, J. Saget, J. Pyun, S. Jia, T. Kowalewski, K. Matyjaszewski, Macromolecules 2003, 36, 8969-8977.

9. M. Kamigaito K. Satoh, Macromolecules 2008, 41, 269-276.

10. W.-F. Kuan, R. Roy, L. Rong, B. S. Hsiao, T. H. Epps III, ACS Macro Lett. 2012, 1, $519-523$.

11. H. Jinnai, T. Kaneko, K. Matsunaga, C. Abetz, V. Abetz, Soft Matter 2009, 5, 20422046.

12. T. Higuchi, H. Sugimori, X. Jiang, S. Hong, K. Matsunaga, T. Kaneko, V. Abetz, A. Takahara, H. Jinnai, Macromolecules 2013, 46, 6991-6997.

13. F. Schacher, J. Yuan, H. G. Schoberth, A. H. E. Müller, Polymer 2010, 51, 2021-2032. 
14. K. Hayashida, T. Dotera, A. Takano, Y. Matsushita, Phys. Rev. Lett. 2007, 98, 195502.

15. Y. Matsushita, K. Hayashida, T. Dotera, A. Takano, J. Phys. Condens. Matter 2011, 23, 284111.

16. A.-C. Shi B. Li, Soft Matter 2013, 9, 1398-1413.

17. H. Yabu, T. Higuchi, H. Jinnai, Soft Matter 2014, 10, 2919-2931.

18. A. Knoll, A. Horvat, K. Lyakhova, G. Krausch, G. Sevink, A. V. Zvelindovsky, R. Magerle, Phys. Rev. Lett. 2002, 89, 035501.

19. T. P. Russell, A. Menelle, S. Anastasiadis, S. Satija, C. Majkrzak, Macromolecules 1991, 24, 6263-6269.

20. N. Koneripalli, N. Singh, R. Levicky, F. S. Bates, P. D. Gallagher, S. K. Satija, Macromolecules 1995, 28, 2897-2904.

21. K. Niihara, H. Sugimori, U. Matsuwaki, F. Hirato, H. Morita, M. Doi, H. Masunaga, S. Sasaki, H. Jinnai, Macromolecules 2008, 41, 9318-9325.

22. P. Dobriyal, H. Xiang, M. Kazuyuki, J.-T. Chen, H. Jinnai, T. P. Russell, Macromolecules 2009, 42, 9082-9088.

23. K. Shin, H. Xiang, S. Moon, T. Kim, T. McCarthy, T. P. Russell, Science 2004, 306, 76.

24. M. Pinna, X. Guo, A. V. Zvelindovsky, J. Chem. Phys. 2009, 131, 214902.

25. A. C. Arsenault, D. A. Rider, N. Tetreault, J. I. L. Chen, N. Coombs, G. A. Ozin, I. Manners, J. Am. Chem. Soc. 2005, 127, 9954-9955.

26. H. Yabu, T. Jinno, K. Koike, T. Higuchi, M. Shimomura, Macromolecules 2011, 44 , $5868-5873$.

27. M. Pinna, S. Hiltl, X. Guo, A. Boeker, A. V. Zvelindovsky, ACS Nano 2010, 4, 28452855 . 
28. E. W. Edwards, M. F. Montague, H. H. Solak, C. J. Hawker, P. F. Nealey, Adv. Mater. 2004, 16, 1315-1319.

29. S. Ji, G. Liu, F. Zheng, G. S. W. Craig, F. J. Himpsel, P. F. Nealey, Adv. Mater. 2008, 20, 3054-3060.

30. R. Dessi, M. Pinna, A. V. Zvelindovsky, Macromolecules 2013, 46, 1923-1931.

31. H. Yabu, T. Higuchi, M. Shimomura, Adv. Mater. 2005, 17, 2062-2065.

32. T. Higuchi, A. Tajima, K. Motoyoshi, H. Yabu, M. Shimomura, Angew. Chem. Int. Ed. 2008, 47, 8044-8046.

33. T. Higuchi, K. Motoyoshi, H. Sugimori, H. Jinnai, H. Yabu, M. Shimomura, Macromol. Rapid Commun. 2010, 31, 1773-1778.

34. T. Higuchi, K. Motoyoshi, H. Sugimori, H. Jinnai, H. Yabu, M. Shimomura, Soft Matter 2012, 8, 3791-3797.

35. J. Fraaije G. Sevink, Macromolecules 2003, 36, 7891-7893.

36. G. J. A. Sevink A. V. Zvelindovsky, Macromolecules 2005, 38, 7502-7513.

37. P. Chen, H. Liang, A.-C. Shi, Macromolecules 2008, 41, 8938-8943.

38. R. Yang, B. Li, A.-C. Shi, Langmuir 2012, 28, 1569-1578.

39. M. Pinna, S. Hiltl, X. Guo, A. Boeker, A. V. Zvelindovsky, ACS Nano 2010, 4, 28452855.

40. B. Yu, B. Li, Q. Jin, D. Ding, A.-C. Shi, Macromolecules 2007, 40, 9133-9142.

41. P. Chi, Z. Wang, B. Li, A.-C. Shi, Langmuir 2011, 27, 11683-11689.

42. B. Yu, J. Deng, B. Li, A.-C. Shi, Soft Matter 2014, 10, 6831-6843.

43. N. Kawase, M. Kato, H. Nishioka, H. Jinnai, Ultramicroscopy 2007, 107, 8-15.

44. P. K. Luther, M. C. Lawrence, R. A. Crowther, Ultramicroscopy 1988, 24, 7-18. 
45. H. Jinnai R. J. Spontak, Polymer 2009, 50, 1067-1087.

46. H. Jinnai, R. J. Spontak, T. Nishi, Macromolecules 2010, 43, 1675-1688.

47. Y. Oono S. Puri, Phys. Rev. A 1988, 38, 434-453.

48. M. Bahiana Y. Oono, Phys. Rev. A 1990, 41, 6763-6771.

49. S. Qi Z.-G. Wang, Phys. Rev. E 1997, 55, 1682-1697.

50. M. Pinna A. V. Zvelindovsky, Eur. Phys. J. B 2012, 85, 210.

51. T. Ohta K. Kawasaki, Macromolecules 1986, 19, 2621-2632.

52. L. Liebler, Macromolecules 1980, 13, 1602-1617.

53. J. Feng E. Ruckenstein, J. Chem. Phys. 2004, 121, 1609-1625.

54. S. R. Ren, I. W. Hamley, G. J. A. Sevink, A. V. Zvelindovsky, J. G. E. M. Fraaije, Macromol. Theory Simul. 2002, 11, 123-127.

55. S. R. Ren I. W. Hamley, Macromolecules 2001, 34, 116-126.

56. M. Pinna, X. Guo, A. V. Zvelindovsky, Polymer 2008, 49, 2797-2800.

57. I. W. Hamley, Macromol Theor Simul 2000, 9, 363-380.

58. M. Pinna, A. V. Zvelindovsky, S. Todd, G. Goldbeck-Wood, J. Chem. Phys. 2006, 125, 154905.

59. H. Yabu, T. Higuchi, K. Ijiro, M. Shimomura, Chaos 2005, 15, 047505. 
Figure 1: A stencil for Laplacian, where NN denotes nearest neighbours, NNN - next-nearest neighbours and NNNN - next-next nearest neighbours neighbours to the point $\mathbf{r}$.

Figure 2: Preparation procedure of PSt-b-PI nanoparticles. In the STEM image, the bright and dark regions correspond to the PSt and PI phases, respectively.

Figure 3: TEM and 3D structures of block copolymer nanoparticles obtained with TEMT. The blue and green phases in 3D images correspond to the PSt and PI phases, respectively. Scale bar: $100 \mathrm{~nm}$.

Figure 4: Plot of area ratios occupied by PI phases at the particle surfaces $\left(S_{\mathrm{PI}}\right)$ as a function of $D / L_{0} . S_{\mathrm{PI}}$ was measured from $3 \mathrm{D}$ data obtained with TEMT.

Figure 5: Cell dynamics simulation of diblock copolymer in spherical confinement for the boundary parameter $\psi_{0}=0.2$. The green and blue blocks correspond to the major (PI) and minor (PSt) phases, respectively, in the experiments. The volume fraction is $f_{\mathrm{PI}}=0.60$.

Figure 6: Cell dynamics simulation of diblock copolymer in spherical confinement for the boundary parameter $\psi_{0}=0.05$. The green and blue blocks correspond to the major (PI) and minor (PSt) phases, respectively, in the experiments. The volume fraction is $f_{\mathrm{PI}}=0.58$. 


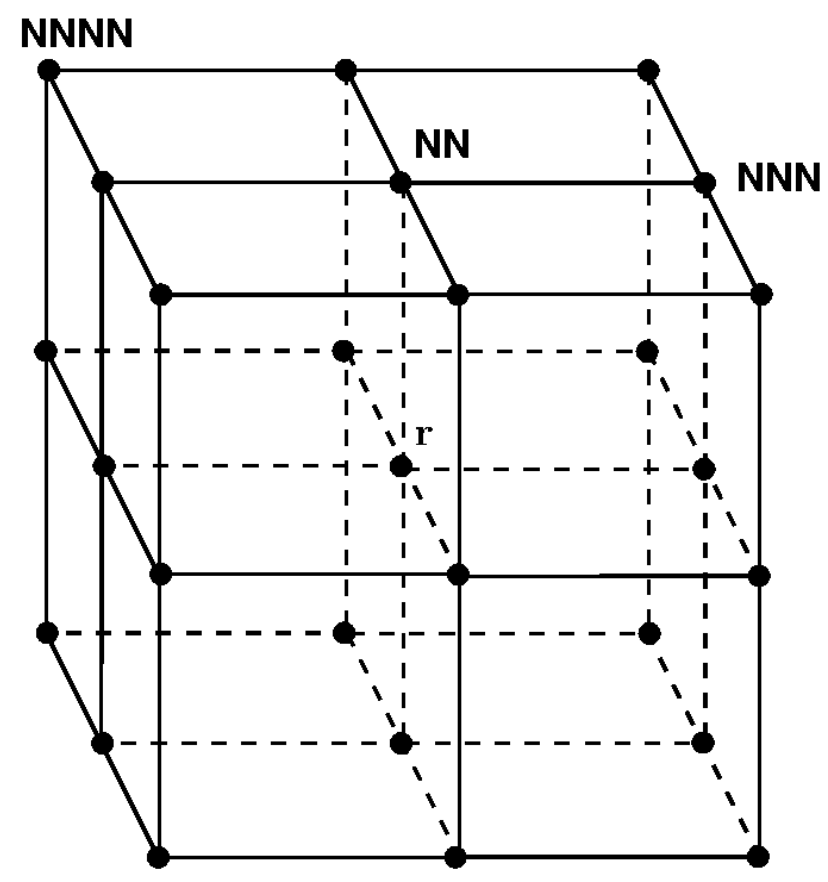

Figure 1

T. Higuchi, M. Pinna, A. V.

Zvelindovsky, H. Jinnai, $H$. Yabu

J. Polym. Sci. B

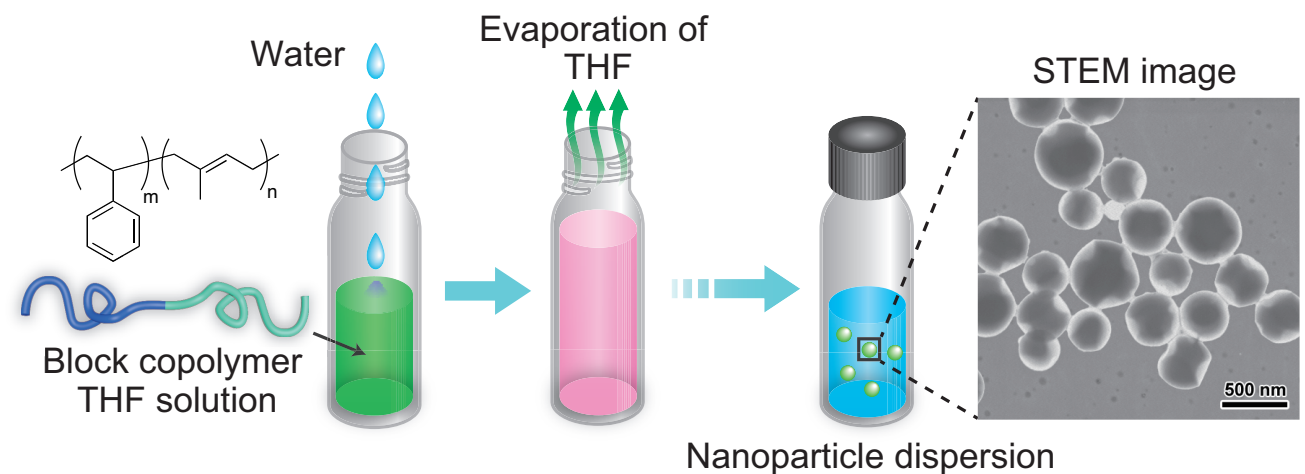

Figure 2

T. Higuchi, M. Pinna, A. V.

Zvelindovsky, H. Jinnai, H.

Yabu

J. Polym. Sci. B 


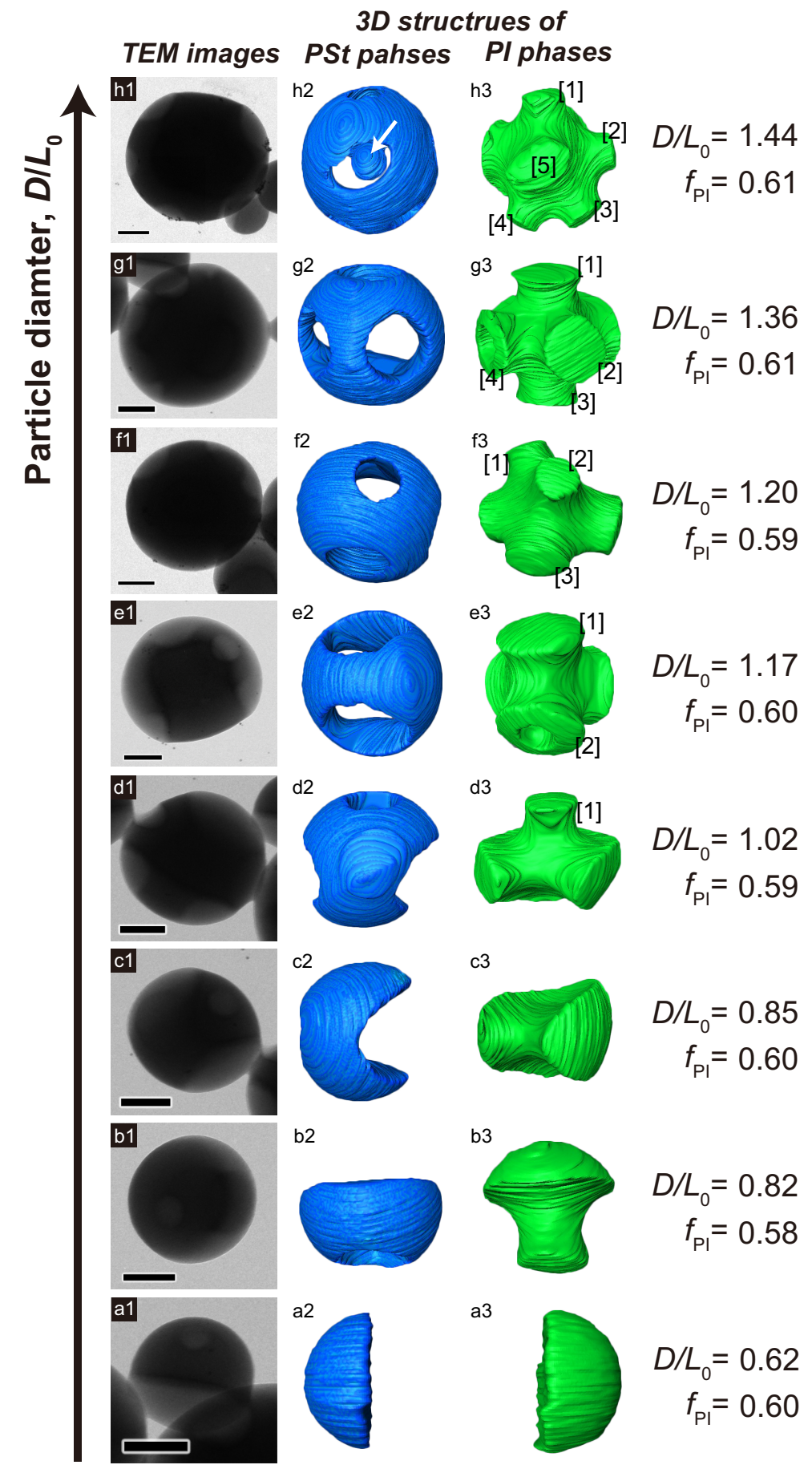

Figure 3

T. Higuchi, M. Pinna, A. V. Zvelindovsky, H. Jinnai, H. Yabu

J. Polym. Sci. B 


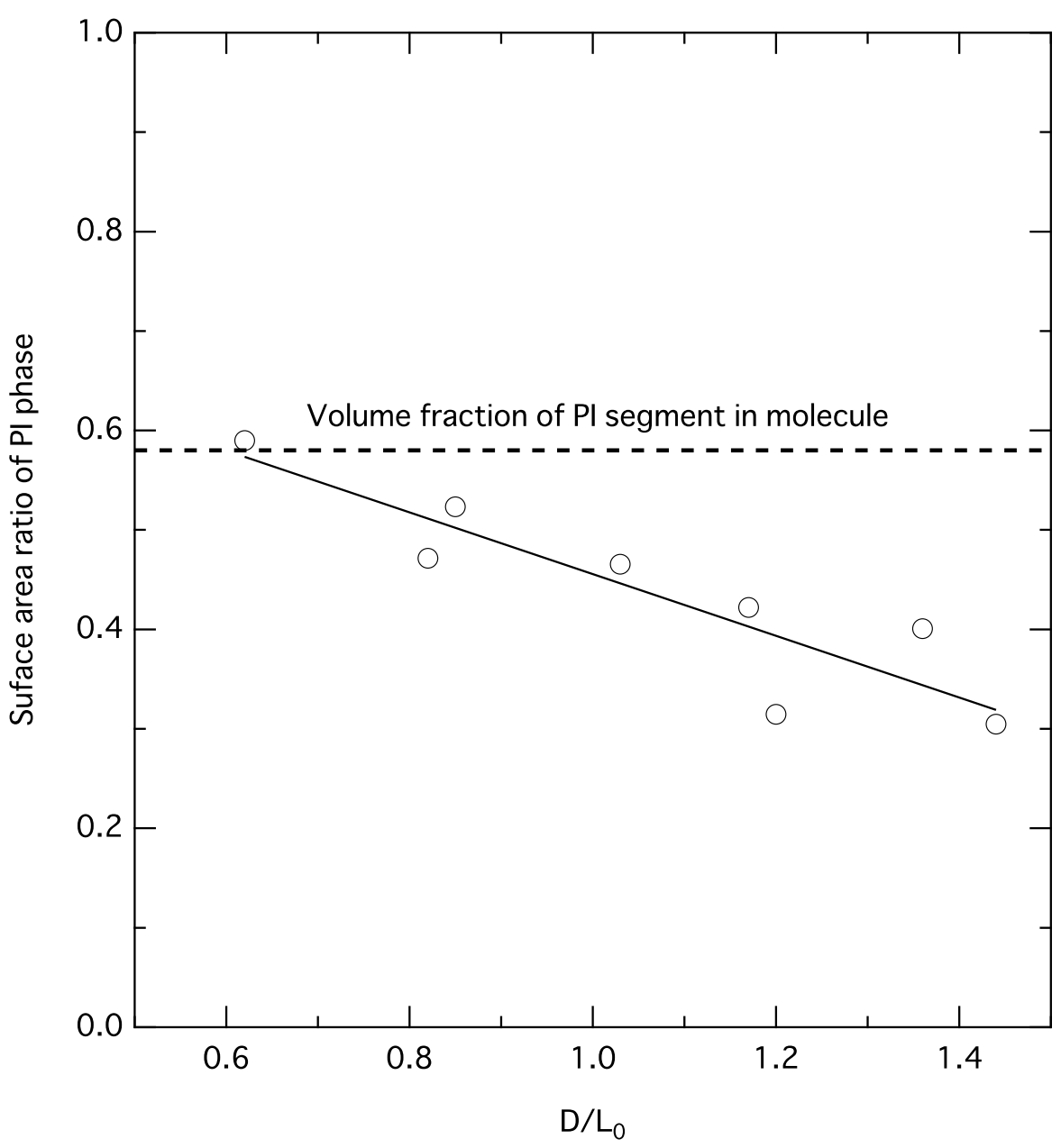

Figure 4

T. Higuchi, M. Pinna, A. V.

Zvelindovsky, H. Jinnai, H.

Yabu

J. Polym. Sci. B 


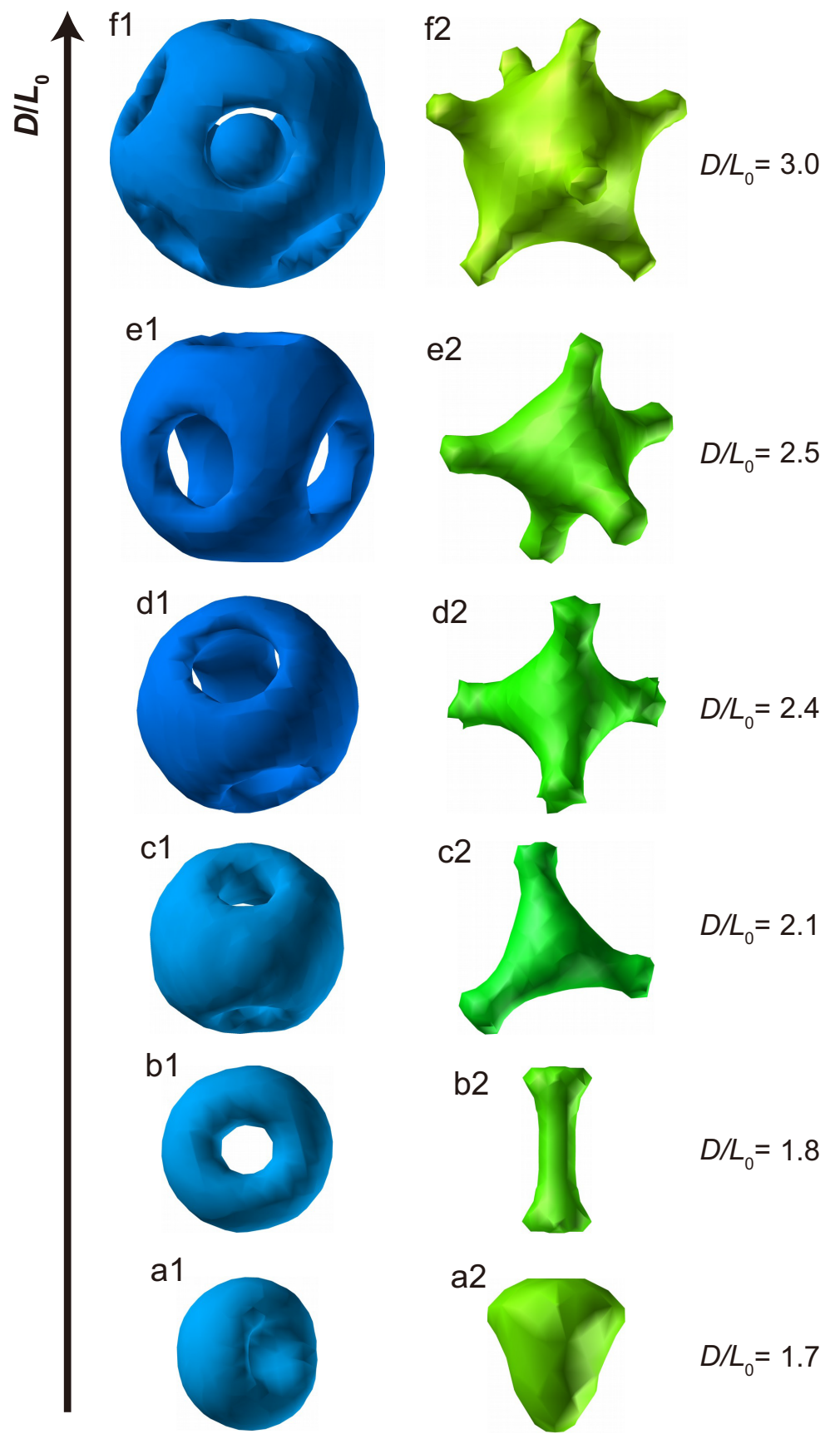

Figure 5

T. Higuchi, M. Pinna, A. V. Zvelindovsky, H. Jinnai, H. Yabu

J. Polym. Sci. B 


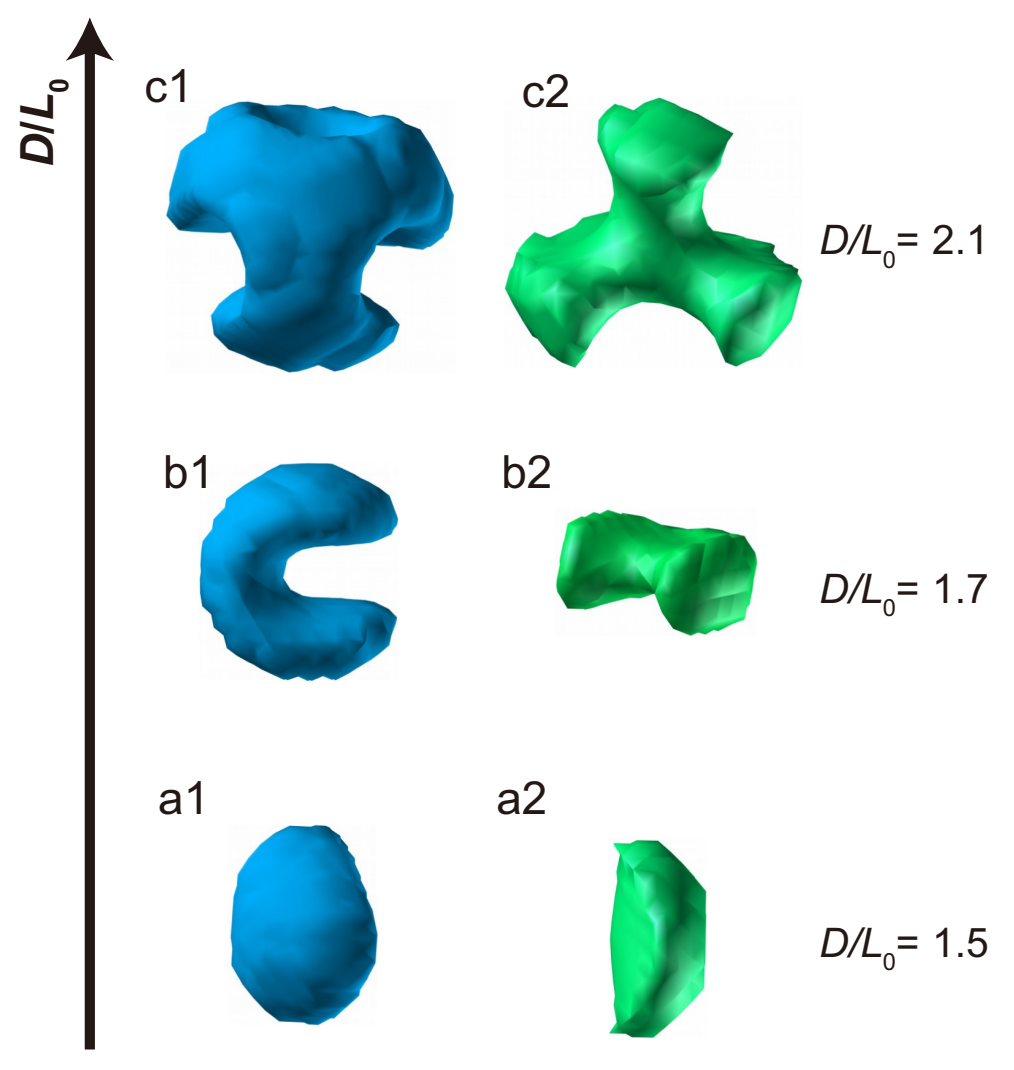

Figure 6

T. Higuchi, M. Pinna, A. V. Zvelindovsky, H. Jinnai, H. Yabu

J. Polym. Sci. B 


\section{Graphical Abstract}

T. Higuchi*, M. Pinna*, A. V. Zvelindovsky, H. Jinnai, H. Yabu*

Multi-pod structures of lamellae-forming diblock copolymers in three-dimensional confinement spaces: experimental observation and computer simulation

The three-dimensional (3D) confinement effect on the microphase-separated structure of a diblock copolymer was investigated both experimentally and theoretically. In conjunction with the experiment and theory, the morphologies of the $3 \mathrm{D}$ confinements were computationally simulated by using some structural parameters extracted from the actual 3D structures obtained with transmission electron microtomography. The simulated structures appear in good agreement with the experimental results.

ToC figure $(50 \mathrm{~mm}$ wide $\times 50 \mathrm{~mm}$ high $)$

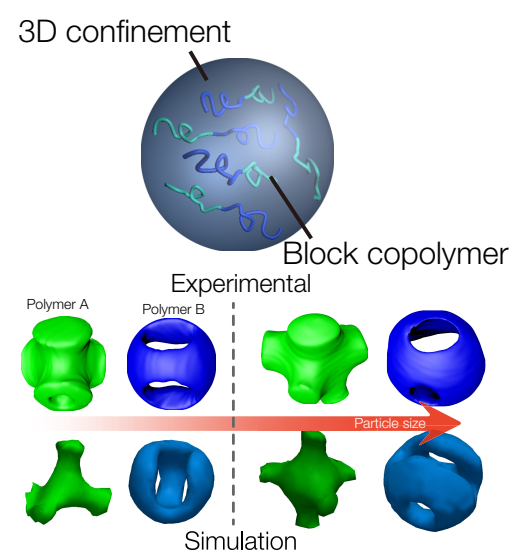

